Human Ethology Bulletin 31 (2016)3: 15-24

Brief Report

\title{
Non-Mammalian Infants DePENDENT ON PARENTAL CARE ELICIT GREATER KINDCHENSCHEMA-RELATED Perceptions and Motivations in Humans
}

\author{
Daniel J. Kruger' ${ }^{1}$ \& Steven A. Miller ${ }^{2}$ \\ ${ }^{1}$ School of Public Health, University of Michigan, Ann Arbor, USA \\ ${ }^{2}$ Department of Psychology, Rosalind Franklin University of Medicine and Science, North \\ Chicago, USA \\ kruger@umich.edu
}

\begin{abstract}
Ethologist Konrad Lorenz (1943) proposed the co-evolution of certain care eliciting characteristics, named Kindchenschema (baby schema), in altricial infants and caregiving responses to these characteristics in parents. Pedomorphic facial characteristics in human infants and adults cue social approach and elicit helping. Lorenz (1943) proposed that Kindchenschema features important for eliciting caregiving are similar across species. This is the first experiment to demonstrate that non-mammalian species dependent on parental care elicit greater Kindchenschema-related perceptions and motivations than infant species in the same class that are not dependent on parental care. Our results complement findings from studies using images of human infants and computer generated/manipulated images as stimuli.
\end{abstract}

Key words: Kindchenschema, parental care, pedomorphism, neoteny, precocial

\section{INTRODUCTION}

Ethologist Konrad Lorenz (1943) proposed the co-evolution of certain care eliciting characteristics in altricial infants and caregiving responses to these characteristics in parents. Lorenz described these characteristics as the Kindchenschema (baby schema). They include large eyes, a large and protruding forehead, a small chin, pudgy lips, and thin, arched brows 
in humans (Alley, 1988). They evoke perceptions of cuteness, and elicit caregiving (Alley, 1981; Glocker, Gur, Langleben, Loughead, Ruparel, \& Sachser, 2009;, Zebrowitz, Brownlow, \& Olson, 1992). Lorenz described similar features in the young of other species when compared to the adults of those species. They are proposed to be an important factor in maintaining caregiving and so the survival of those young where parental caregiving is necessary for that survival. (Bowlby, 1969; Eibl-Eibesfeldt, 1989). Lorenz' (1943) proposal has generated a considerable amount of research, yet gaps remain in the empirical validation of his model.

The term pedomorphic is used when adult human faces exhibit these features. Such faces tend to elicit attributions of being more warm, honest, and sincere but also naive and physically weak (Berry, 1991; McArthur \& Apatow, 1984). Keating, Randall, Kendrick, and Gutshall (2003) digitally enlarged eyes and lips on adult faces, mimicking pedomorphism, and students rated the resulting faces as more submissive, weak, naive, feminine, compassionate, and honest than the original images. In addition, returns of "lost" letters (see Milgram, Mann, \& Harter, 1965) were generally higher when the resume contained a pedomorphed image than an original image. Infant faces that are digitally manipulated to increase Kindchenschema features produce greater activation in the nucleus accumbens of female viewers who have never had children (Glocker, Langleben, Ruparel, Loughead, Valdez, Griffin, Sachse, \& Gur, 2009). The nucleus accumbens is a brain area that mediates reward processing and appetitive motivation (Malenka, Nestler, \& Hyman, 2009).

Existing studies on Kindchenschema use images of human infants as stimuli, some computer generated or manipulated, but none with actual images of infants from other species. No previous research has examined human reactions to naturalistic images of infants from non-human, especially non-mammalian, species. If there is a similarity between the defining features of infant Kindchenschema and caretaking reactions across species, as Lorenz (1943) proposed, human caretaking intentions towards the young of avian and reptile species should be greater the more altricial the species. We predicted that human Kindchenschema-related perceptions and motivations would be greater for images of nonmammalian infants dependent on parental care for their survival compared to reactions for images of infants that are completely independent at hatching. The Kindchenschema reaction complex includes perceptions of cuteness, assumptions of helplessness, and desires to provide caregiving.

Kindchenschema characteristics in mammals are associated with a cluster of traits including adult attachment, paternal care, small social groups, and small body sizes (Fraley, Brumbaugh, \& Marks, 2005). All mammal species exhibit maternal care and food provisioning, with varying degrees of paternal care and provisioning, including none (Low, 2000). Gill (1995) classifies birds along a continuum from altricial to superprecocial, ranging from least to most physiologically mature at hatching, and from most to least dependent on parents. 
- Altricial

- Semi-altricial

- Semi-precocial

- Sub-precocial

- Precocial

- Superprecocial

Infants in many avian and reptile species are truly precocial, these "superprecocial" species do not exhibit parental care, as infants are completely independent at hatching (Gill, 1995). Superprecocial bird infants leave the nest within two days of hatching and are capable of feeding themselves. The newly hatched superprecocial megapode chicks dig themselves out of the nest mound without parental assistance, have full flight feathers, and can fly on the same day they hatch. Semiprecocial bird infants are somewhat independent; they can walk and often swim, and are capable of leaving the nest soon after hatching. However, semiprecocial bird infants are dependent on parents for food and remain nearby the nest site. They hatch with their eyes open and are covered with down.

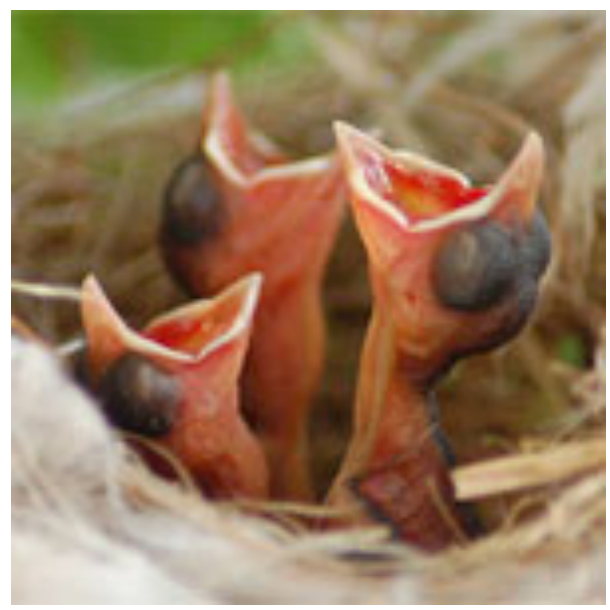

Figure 1. Altricial Bird Infants. Modified from:

http://free-images.gatag.net/images/201109200600.jpg

The characteristics of altricial bird infants are so extreme that they may not be suitable for testing our hypothesis, as they are naked, blind (their eyes are closed), and helpless at hatching. It is possible that there is a different mechanism, or at least different visual properties as elicitors of parental care, in altricial bird species. Rather than viewing the Kindchenschema characteristics associated with semiprecocial bird infants, parents of recently hatched altricial infants see the interiors of gaping mouths when returning to the nest (See Figure 1). This visual mechanism has been exploited via the supernormal stimuli 
of brood parasites such as the cuckoo. Thus, we utilize images of semiprecocial bird infants and superprecocial bird infants, selected based on classifications of the degree of parental care (e.g., Gill, 1995), in our experimental conditions. Crocodiles and their relatives are the only reptiles that tend to both eggs and hatchlings (Ehrlich, Dobkin, \& Wheye, 1988). Thus, a crocodile infant would be a suitable comparison for another infant reptile with a similar body form (e.g., a lizard).

\section{METHOD}

We searched Google Images for appropriate stimuli, pictures of species specifically listed by Gill (1995) as being superprecocial or semiprecocial. We used the search terms "hatchling," "chick," and "infant" along with the name of a superprecocial or semiprecocial bird species (e.g., "tern") or the terms "crocodile" or "lizard." We selected images that fit the following criteria:

1) A high-resolution color picture with no visual alterations

2) Depicting at least $80 \%$ of the animal

3) Including its entire head

4) No portion of the animal outside of the image frame

5) In its natural environment

6) By itself with no other animals visible

We utilized the first image appearing that matched all six of these criteria. Most webpages hosting these images indicated that the infants had recently hatched. We cropped images to remove background (and any text) and resized them to maximize similarity across images.

Table 1. Species included in infant image stimuli

\section{Superprecocial birds}

Australian brush turkey, Alectura lathami

Tabon Megapode, Megapodius cumingii

Black-headed duck, Heteronetta atricapilla
Semiprecocial birds

Least tern, Sternula antillarum

Ring billed gull, Larus delawarensis

Emperor Penguin, Aptenodytes forsteri

\begin{tabular}{lll}
\hline $\begin{array}{l}\text { Superprecocial reptile } \\
\text { California Alligator Lizard, Elgaria } \\
\begin{array}{l}\text { multicarinata multicarinata } \\
\hline\end{array}\end{array} \quad$ Dwarf crocodile, Osteolaemus tetraspis \\
\hline
\end{tabular}

We included these images in an Internet-based survey on the Qualtrics ${ }^{\mathrm{TM}}$ platform. Ethnically diverse undergraduates $(N=172 ; 50 \%$ female, $M$ age $=19, S D$ age $=1)$ at a large public university in the Midwestern USA completed anonymous on-line surveys at their 
convenience. Participants were asked "What ethnicities do/did your grandparents belong to?" and could check all categories that applied. Proportionally these were:

- $49 \%$ Western European

- $41 \%$ Eastern European

- $10 \%$ East Asian

- $6 \%$ South Asian

- $6 \%$ African American

- $5 \%$ Latino/a/Hispanic

- $4 \%$ Arab/Middle-Eastern

- $3 \%$ Native American/Alaskan native

- One Pacific Islander

- $5 \%$ Other

The survey program displayed images of each animal, presented in a randomized order, along with survey items on separate pages. Participants used a sliding graphic scale ranging from 0 to $100 \%$, and initially set at $50 \%$, to rate each item. Scale values were displayed and could be modified until participants advanced to the next page. Participants first rated each animal on perceptions of altricial characteristics and attractiveness: Attractive, cute, helpless, independent, mature, and young, presented in randomized order. Scores for independent and mature were reverse-coded. Participants then completed caregiving intention items:

- To what extent would you like to hold or pet this animal?

- How likely would this animal be able to survive on its own?

- If you were working out in the field and found this animal wandering around by itself, how likely would you be to adopt it at least temporarily to make sure that it survived?

Scores for the survival item were reverse-coded, and values for the nine items were averaged to create a scale score representing the Kindchenschema reaction complex (K-complex). Participants then read the item, "Do you recognize this animal?" Those who responded "yes" or "maybe" were asked "What is it? What do you know about it?" and were provided an open-ended text response field. Participants could answer an open-ended text response field for general feedback, "Do you have any comments about this survey?"

As each participant completed items on all eight animal infants, responses by animal infant are nested within individuals. Thus, we conducted a Multi-Level-Model (MLM) with a random effect of person to model the best covariance structure for correlations of the dependent measure within person. The model examines whether there were significant 
effects of parental dependency and class (whether the infant animal is a reptile or bird), and whether there was a significant interaction between parental dependency and class on the Kcomplex.

\section{RESULTS}

Multilevel modeling was used to examine effects; models were built including effects of participant, stimuli, and interactions (See Table 2). We tested seven models with all possible combinations of effects. The model (7) with two main effects, interaction, and random intercept fit the data best. As expected, images of infants dependent on parental care elicited greater Kindchenschema-related perceptions and motivations, $\mathrm{F}(1,1201)=2801.36$, $\mathrm{p}<$ .001. Kindchenschema-related perceptions and motivations were also higher for avian images than for reptile images, $\mathrm{F}(1,1201)=579.73, \mathrm{p}<.001$, and the effect of parental dependency was stronger for avian images than for reptile images, $F(1,1201)=38.43$, $\mathrm{p}<$ .001 (See Figure 2). The intra-class correlation ( $\rho$; i.e., the amount of variance in outcome attributable to nesting of data within subjects) for the restricted maximum likelihood estimate of the best fitting model was 0.28 .

Table 2. Parameter estimates for seven models examining effects of parental dependency, reptile (vs. avian), and parental dependency $x$ reptile (vs. avian) on K-Complex

\begin{tabular}{|c|c|c|c|c|c|c|c|c|}
\hline Effect & Parameter & Model 1 & Model 2 & Model 3 & Model 4 & Model 5 & Model 6 & Model 7 \\
\hline \multicolumn{9}{|l|}{ Fixed Component } \\
\hline Intercept & \multirow{4}{*}{$\begin{array}{l}\hat{\gamma}_{00} \\
\hat{\gamma}_{01} \\
\hat{\gamma}_{02} \\
\hat{\gamma}_{03}\end{array}$} & \multirow[t]{4}{*}{46.52} & \multirow[t]{4}{*}{46.52} & 58.31 & \multirow[t]{2}{*}{32.22} & 44.02 & 39.98 & 39.98 \\
\hline Parental Dependency & & & & -23.59 & & -23.59 & -15.52 & -15.52 \\
\hline Reptile & & & & & \multirow[t]{2}{*}{19.06} & 19.06 & 24.44 & 24.44 \\
\hline Parent Dependency x Reptile & & & & & & & -10.76 & -10.76 \\
\hline \multicolumn{9}{|c|}{ Variance of Random Component } \\
\hline Intercept & \multirow[b]{2}{*}{$\begin{array}{l}\hat{\tau}_{00} \\
\hat{\sigma}^{2}\end{array}$} & & 43.74 & 63.61 & 53.47 & 73.34 & 73.34 & 74.63 \\
\hline Residual & & 480.70 & 436.96 & 277.95 & 359.13 & 200.12 & 193.92 & 194.40 \\
\hline \multicolumn{9}{|l|}{ Model Misfit Information } \\
\hline Deviance (-2LL) & & 12402.04 & 12372.95 & 11827.26 & 12136.79 & 11432.74 & 11394.82 & 11387.78 \\
\hline AIC & & 12406.04 & 12378.95 & 11835.26 & 12144.79 & 11442.74 & 11406.82 & 11391.78 \\
\hline BIC & & 12416.50 & 12394.63 & 11856.16 & 12165.70 & 11468.87 & 11437.18 & 11401.22 \\
\hline
\end{tabular}

Note: All effects are significant at $\mathrm{p}<.001$. Models $1-6$ are estimated using maximum likelihood to allow $-2 \mathrm{LL}$ comparison. Model 7 is estimated using restricted maximum likelihood, to provide more accurate variance components. 


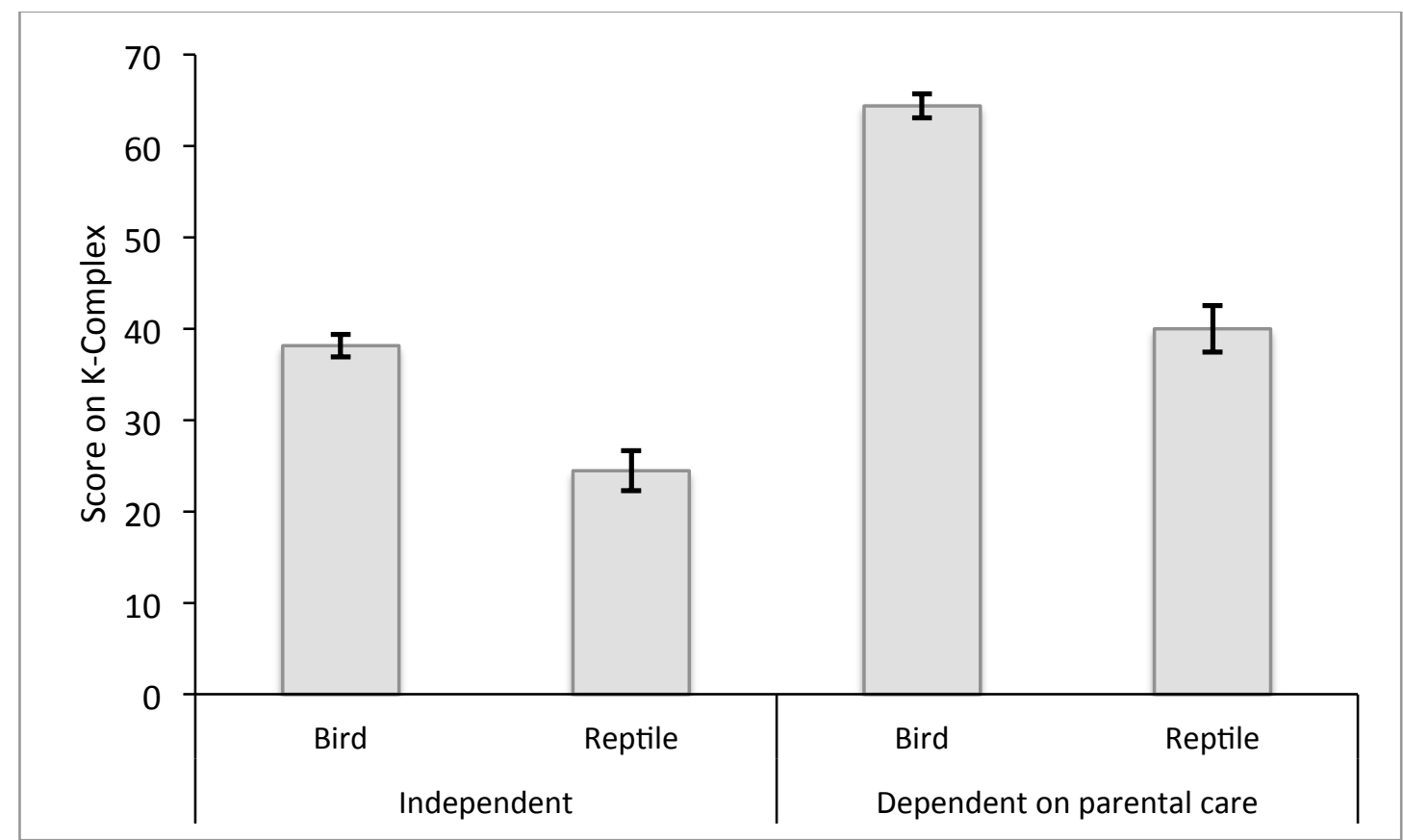

Figure 2. Best Linear Unbiased Estimate (BLUE) of K-Complex by parental dependency and class with $95 \%$ Confidence Intervals

\section{DISCUSSION}

The results confirmed our prediction that Kindchenschema-related perceptions and motivations in humans would be elicited more so by images of non-human animal infants that are dependent on parental care, compared to images of independent superprecocial infants. This supports Lorenz' (1943) proposal that there are cross species similarities in the features of Kindchenschema, at least as perceived by humans. We demonstrate this pattern for both avian and reptile stimuli, and note that the effect was stronger for avian stimuli. Our cross-species and cross-class results provide support for Lorenz' (1943) model, and complement existing support from studies using real images of human infants as well as facial images that have been transformed though computer software to exaggerate or diminish pedomorphic characteristics. We used actual images of newly hatched animal infants in their natural environments.

\section{Limitations}

Our participants are undergraduates at a university in the Midwestern USA. Although they are ethnically diverse (73\% indicated some non-Western European ancestry, 11 were born outside of North America), undergraduate samples from North American universities have been criticized as not being representative of the entire human population (e.g., Henrich, Heine, \& Norenzayan, 2010). We do not know how our participants' responses differ from 
other populations, especially those who have a greater extent of experiences with the natural world and/or contact with non-domesticated animals. Replications of the study in diverse populations would bolster the case that these phenomena are human universals.

We selected our stimuli from images available to the public over the Internet. These images were taken by different photographers, with different cameras, using different settings, in different environments. They varied in viewing angle, lighting, background, stance and posture, and other potentially relevant properties. Although these images were not standardized (as computer generated images could be), we do not expect that the images are biased with respect to the experimental hypothesis. The images were freely shared by professional and amateur photographers, who are likely presenting what they consider high quality work, and of course were unaware of the experimental hypothesis. Rather than using computer software to geometrically assess or manipulate Kindchenschema, we selected images from species that theoretically should differ in the degree of Kindchenschema features. These limitations are a trade-off for the use of stimuli representing natural, real world animal infants. Computer generated images can provide greater consistency and experimental control; however they can also be unrealistic, depicting supernormal or unnatural stimuli outside of the actual range of attributes in the natural world.

We anticipated that some animals would be more familiar to participants than others would. Familiarity is associated with liking (Zajonc, 2001), and thus may influence study variables. Participants indicated highest familiarity with the penguin, consistent with its frequent media appearances. However, superprecocial animals were the second and third most familiar, and very few participants identified any of the other animals.

Finally, we assessed perceptions and intentions (i.e., hypothetical caregiving reactions), as is the case with nearly all of the previous studies in this area. Studies including actual behavioral reactions (e.g., Zebrowitz, Brownlow, \& Olson, 1992) are rare and involve human infants. Behavioral reaction studies to actual animal infants would require the capture and containment of such animals. Such studies would be easier to conduct with species more commonly available for laboratory studies or possibly utilizing animals housed in zoos or wildlife rehabilitation sanctuaries.

\section{Conclusion}

This study broadens the empirical support for Lorenz' (1943) Kindchenschema theory. Our results may have implications beyond biological theory, as they may help explain differential support for conservation efforts based on the physical appearance of species (see Estren, 2012). Species with greater degrees of Kindchenschema may be more effective in eliciting human compassion and actions promoting ecological conservation. 


\section{REFERENCES}

Alley, T.R. (1981). Head shape and the perception of cuteness. Developmental Psychology, 17, 650654. DOI

Alley, T. R. (1988). The effects of growth and aging on facial aesthetics. In T. R. Alley (Ed.), Social and applied aspects of perceiving faces (pp. 51-62). Hillsdale, NJ: Erlbaum.

Berry, D. S. (1991). Attractive faces are not all created equal: Joint effects of facial babyishness and attractiveness on social perception. Personality and Social Psychology Bulletin, 17, 523-533. DOI

Cohen, J. (1988). Statistical power analysis for the behavioral sciences (2nd ed.). New York: Academic Press. DOI

Ehrlich, P., Dobkin, D., \& Wheye, D. (1988). The Birder's Handbook. New York, NY: Simon \& Schuster.

Eibl-Eibesfeldt, I. (1989). Human Ethology. New York: De Gruyter.

Estren, M.J. (2012). The neoteny barrier: Seeking respect for the non-cute. Journal of Animal Ethics, 2, 6-11. DOI

Fraley, R. C., Brumbaugh, C. C., \& Marks, M. J. (2005). The evolution and function of adult attachment: A comparative and phylogenetic analysis. Journal of Personality and Social Psychology, 89, 731-746. DOI

Gill, F.B. (1995). Ornithology, 2nd ed. New York, NY: W.H. Freeman and Co.

Glocker, M.L., Gur, R. C., Langleben, D. D., Loughead, J. W., Ruparel, K., Sachser, N. (2009). Baby schema in infant faces induces cuteness perception and motivation for caretaking in adults. Ethology, 115, 257-263. DOI

Glocker, M.L., Langleben, D.D., Ruparel, K., Loughead, J.W., Valdez, J.N., Griffin, M.D., Sachser, N., Gur, R.C. 2009: Baby schema modulates the brain reward system in nulliparous women. Proceedings of the National Academy of Sciences of the USA, 106, 9115-9119. DOI

Henrich, J., Heine, S. J., \& Norenzayan A. (2010). The weirdest people in the world? Behavioral and Brain Sciences, 33, 61-83. DOI

Keating, C.F., Randall, D.W., Kendrick, T., \& Gutshall, K.A. (2003). Do babyfaced adults receive more help? The (cross-cultural) case of the lost resume. Journal of Nonverbal Behavior, 27, 89109. DOI

Lorenz, K. (1943). Die angeborenen Formen möglicher Erfahrung. Zeitschrift für Tierpsychologie, 5, 234-409. DOI

Lorenz, K. (1971). Studies in Human and Animal Behavior. Cambridge, MA: Harvard University Press.

Low, B. S. (2000). Why Sex Matters: A Darwinian look at human behavior. Princeton, NJ: Princeton University Press. DOI 
Malenka, R.C., Nestler, E.J., \& Hyman, S.E. (2009). Neural and neuroendocrine control of the internal milieu. In A. Sydor \& R. Y. Brown (eds.), Molecular Neuropharmacology: A Foundation for Clinical Neuroscience (2nd ed.). New York: McGraw-Hill Medical.

McArthur, L. Z., \& Apatow, K. (1984). Impressions of baby-faced adults. Social Cognition, 2, 315342. DOI

Milgram, S., Mann, L., \& Harter, S. (1965). The lost-letter technique: A tool of social research. Public Opinion Quarterly, 29, 437-438. DOI

Zajonc, R.B. (2001). Mere exposure: A gateway to the subliminal. Current Directions in Psychological Science, 10, 224-228. DOI

Zebrowitz, L. A., Brownlow, S., \& Olson, K. (1992). Baby talk to the babyfaced. Journal of Nonverbal Behavior, 16, 143-158. DOI 